\title{
Density gradient theory combined with the PC-SAFT equation of state used for modeling the surface tension of associating systems
}

\author{
Václav Vinš $\check{~}^{1, a}$, Barbora Planková ${ }^{1}$, Jan Hrubý ${ }^{1}$ and David Celný ${ }^{1}$ \\ ${ }^{1}$ Institute of Thermomechanics AS CR, v. v. i., Dolejškova 1402, 18200 Prague 8, Czech Republic
}

\begin{abstract}
The density gradient theory (GT) combined with a SAFT-type (Statistical Associating Fluid Theory) equation of state has been used for modeling the surface tension of associating fluids represented by a series of six alkanols ranging from methanol to 1-pentanol. The effect of nonzero dipole moment of the selected alkanols on the predicted surface tension was investigated in this study. Results of the GT + non-polar Perturbed Chain (PC) SAFT equation of state were compared to predictions of GT combined with the PCpolar-SAFT, i.e. PCP-SAFT, equation. Both GT + PC-SAFT and GT + PCP-SAFT give reasonable prediction of the surface tension for pure alkanols. Results of both models are comparable as no significant difference in the modeled saturation properties and in the predicted surface tension using GT was found. Consideration of dipolar molecules of selected alkanols using PCP-SAFT had only minor effect on the predicted properties compared to the non-polar PC-SAFT model.
\end{abstract}

\section{Introduction}

The surface tension of associating fluids, e.g., water, alkanols, amines, plays an important role in many environmental processes and engineering applications such as nucleation, flow through porous media, and distillation. Modeling of surface properties of the these systems is a rather difficult task due to the complicated surface tension dependence on temperature having typically more than one inflection point.

In this study, the Cahn-Hilliard density gradient theory (GT) [1] combined with the physically based equation of state (EoS) are used to model the surface tension for several associating systems. The original Perturbed Chain Statistical Associating Fluid Theory (PC-SAFT) [2] has been extended by the association and the polar interactions between molecules. A set of six various light alkanols, ranging from methanol to 1-pentanol, has been chosen to examine the predictive ability of the GT + PC-SAFT EoS to model surface tension of associating polar fluids. The results for GT combined with the original PC-SAFT EoS with neglected polarity of alcohol molecules are compared to the GT + PC-SAFT model extended by the polar interactions.

\section{PC-SAFT equation of state}

PC-SAFT belongs to family of the SAFT-type equations of state which - thanks to the reasonable physical basis represent valuable models laying between the classical,

\footnotetext{
a Corresponding author: vins@it.cas.cz
}

e.g., cubic, EoSs and approaches of the molecular simulations. Consequently, SAFT-type EoSs have been successfully used in a large variety of engineering and scientific applications during the last twenty years [3].

The PC-SAFT equation uses hard-chains instead of simple hard spheres as the reference fluid. The PC-SAFT EoS can be relatively easily applied to simple fluids such as $n$-alkanes, gases or non-polar halogenated hydrocarbons [2,4,5]. Significantly better prediction of thermophysical properties and phase equilibria can be achieved for these systems compared to classical cubic EoSs over wide temperature, pressure, and composition ranges. The PC-SAFT EoS can also be used for modeling more complicated systems such as polymers [6], polar substances $[7,8]$, or associating fluids, e.g., mixtures with alkanols [9], or hydrogen sulfide [10].

In the original PC-SAFT EoS [2], the Helmholtz energy $F$ of the fluid system is given by the ideal gas contribution $F_{\text {id }}$ and the residual part $F_{\text {res }}$ which is defined as a sum of the hard chain contribution $F_{\mathrm{hc}}$ and the perturbation contribution $F_{\text {disp. }}$.

$$
F\left[\mathrm{~J} \cdot \mathrm{mol}^{-1}\right]=F_{\text {id }}+F_{\text {res }}=F_{\text {id }}+F_{\text {hc }}+F_{\text {disp }}
$$

Equation (1) represents a good description for the normal fluids with chemical bonds and basic van der Waals attractions. In PC-SAFT, the hard chain contribution $F_{\mathrm{hc}}$ and the perturbation contribution $F_{\text {disp }}$ can be determined from three molecular parameters [2]; namely segment number $m$, segment diameter $\sigma$, and 
energy parameter $\varepsilon / k_{\mathrm{B}}$. Values for these parameters are usually found by fitting the PC-SAFT equation to the data for the vapor pressure and the saturated liquid density of the modeled fluid.

Unlike standard cubic EoSs, SAFT-type equations can also model other bond-types with bond strengths between the van der Waals attractions and the chemical bonds, e.g., hydrogen bonding or Coulombic forces. A more general expression for the Helmholtz energy in PC-SAFT is given by equation (2)

$$
F=F_{\text {id }}+F_{\text {hc }}+F_{\text {disp }}+F_{\text {as }}+F_{\text {pol }}+\ldots,
$$

where $F_{\text {as }}$ and $F_{\mathrm{pol}}$ stands for association bonds [11] and polar interactions [7], respectively.

\subsection{Association contribution}

Contribution to the Helmholtz energy due to the site-site interactions between molecules or segments - like the hydrogen bonding - can be determined as follows [12-14]

$$
f_{\text {as }}=\frac{F_{\text {as }}}{R T}=\sum_{i=1}^{\operatorname{comp}}\left[\sum_{\mathrm{A}_{i}}^{\operatorname{sites}(i)}\left(\ln X^{\mathrm{A}_{i}}-\frac{X^{\mathrm{A}_{i}}}{2}\right)+\frac{1}{2} M_{i}\right],
$$

where $X^{A_{i}}$ is the mole fraction of molecules $i$ not bonded at site $\mathrm{A}_{i}$ and $M_{i}$ denotes number of associating sites on molecule $i$. $X^{A_{i}}$ can be obtained as follows:

$$
X^{\mathrm{A}_{i}}=\left(1+\sum_{j}^{\mathrm{N}_{\mathrm{comp}}} \sum_{\mathrm{B}_{j}}^{\mathrm{N}_{\text {sites }(j)}} \rho_{j}^{\prime} X^{\mathrm{B}_{j}} \Delta^{\mathrm{A}_{i} \mathrm{~B}_{j}}\right)^{-1},
$$

where $\rho_{j}^{\prime}\left[\AA^{-3}\right]$ is the number density of component $j$

$$
\rho_{j}^{\prime}=x_{j} \rho_{\text {mix }}^{\prime}=x_{j} \frac{N_{\mathrm{AV}}}{10^{30}} \rho_{\text {mix }}\left[\mathrm{mol} \cdot \mathrm{m}^{-3}\right] .
$$

$\Delta^{\mathrm{A}_{i} \mathrm{~B}_{j}}$ is the association strength between sites $\mathrm{A}_{i}$ and $\mathrm{B}_{j}$.

$$
\Delta^{\mathrm{A}_{i} \mathrm{~B}_{j}}=g_{i j}^{\mathrm{hs}}\left[\exp \left(\frac{\varepsilon^{\mathrm{A}_{i} \mathrm{~B}_{j}}}{k_{\mathrm{B}} T}\right)-1\right]\left(\sigma_{i j}\right)^{3} \kappa^{\mathrm{A}_{i} \mathrm{~B}_{j}}
$$

In equation (6), $g_{i j}^{\text {hs }}$ denotes the radial distribution function for the reference hard sphere fluid [2]. $\varepsilon^{\mathrm{A}_{i} \mathrm{~B}_{j}}$ and $\kappa^{\mathrm{A}_{i} \mathrm{~B}_{j}}$ are the association energy and the volume parameters, respectively. Values for $\varepsilon^{\mathrm{AB}}$ and $\kappa^{\mathrm{AB}}$ are similarly as other molecular parameters $\left(m, \sigma\right.$, and $\left.\varepsilon / k_{\mathrm{B}}\right)$ correlated to the available saturation data. Mixture parameters $\varepsilon^{\mathrm{A}_{i} \mathrm{~B}_{j}}$ and $\kappa^{\mathrm{A}_{i} \mathrm{~B}_{j}}$ can be obtained from simple combining rules [11] using pure fluid parameters $\varepsilon^{\mathrm{AB}}$ and $\kappa^{\mathrm{AB}}$.

We note that in equation (6) the temperature independent segment diameter $\sigma_{i j}$ shall be used $[13,14]$ instead of temperature dependent segment diameter $d_{i j}$ as mentioned in the work of Chapman et al. [12].

An overview of various types of bonding in the real associating fluids was, for example, given by Huang and Radosz [13]. Light alcohols considered in this study can be modeled by the so-called $2 \mathrm{~B}$ association model typical of two different associating sites A (hydrogen site) and B (electron pair site on the oxygen atom) on one molecule $\left(M_{i}=2\right)$

\subsection{Polar contribution}

The polar contribution in the residual Helmholtz energy is given as a sum of the quadrupolar [7], dipolar [15] and the combined dipolar-quadrupolar [16] interactions.

$$
F_{\mathrm{pol}}=F_{\mathrm{Q}}+F_{\mathrm{D}}+F_{\mathrm{DQ}}
$$

Gross and Vrabec call PC-SAFT extended by the polar interactions as PC-Polar-SAFT, i.e. PCP-SAFT. The same notation is used in this study to distinguish between PC-SAFT without polar term and PCP-SAFT including the polar interactions.

In PCP-SAFT EoS, no additional molecular parameter for the polar interactions has to be adjusted to the saturation data since the literature values for dipole moment $\mu[\mathrm{D}]$ and quadrupolar moment $Q[\mathrm{D} \AA]$ can be used.

\subsection{PC-SAFT parameters for associating fluids}

The PC-SAFT parameters determined by Gross and Sadowski [11] were used for the selected alcohols when neglecting dipolar moments of the alcohol molecules.

The five molecular parameters for PCP-SAFT parameters were correlated to the available reference data for the vapor pressure and the saturated liquid density.

\begin{tabular}{|c|c|c|c|c|c|c|c|c|c|c|c|c|c|}
\hline Substance & $\begin{array}{l}\mathrm{m} \\
{[-]}\end{array}$ & $\begin{array}{c}\sigma \\
{[\AA]}\end{array}$ & $\begin{array}{l}\varepsilon / k_{\mathrm{B}} \\
{[\mathrm{K}]}\end{array}$ & $\begin{array}{c}\kappa^{\mathrm{AB}} \\
{[-]}\end{array}$ & $\begin{array}{c}\varepsilon^{\mathrm{AB} / k_{\mathrm{B}}} \\
{[\mathrm{K}]}\end{array}$ & $\begin{array}{c}\mu \\
{[\mathrm{D}]}\end{array}$ & $\begin{array}{c}A D(p) \\
{[\%]}\end{array}$ & $\begin{array}{c}A D\left(\rho_{\mathrm{L}}\right) \\
{[\%]}\end{array}$ & $\begin{array}{c}S D(p) \\
{[\%]}\end{array}$ & $\begin{array}{c}S D\left(\rho_{\mathrm{L}}\right) \\
{[\%]}\end{array}$ & $\begin{array}{l}T_{\min } \\
{[\mathrm{K}]}\end{array}$ & $\begin{array}{l}T_{\max } \\
{[\mathrm{K}]}\end{array}$ & Ref. \\
\hline methanol & 2.0653 & 2.9398 & 178.3 & 0.0671 & 2441.9 & 1.70 & -0.587 & -0.031 & 1.394 & 0.314 & 176 & 512 & [18] \\
\hline ethanol & 2.5840 & 3.0889 & 192.0 & 0.0417 & 2488.0 & 1.70 & 0.119 & 0.007 & 0.287 & 0.164 & 260 & 512 & [18] \\
\hline 1-propanol & 2.7995 & 3.3229 & 238.5 & 0.0134 & 2324.7 & 1.55 & 0.175 & 0.026 & 0.711 & 0.543 & 195 & 536 & [19] \\
\hline 2-propanol & 3.1969 & 3.1656 & 204.4 & 0.0306 & 2169.6 & 1.58 & 0.295 & 0.037 & 0.880 & 0.714 & 185 & 508 & [19] \\
\hline 1-butanol & 2.3838 & 3.7933 & 275.5 & 0.0046 & 2678.0 & 1.66 & 0.223 & 0.043 & 0.887 & 0.870 & 225 & 563 & [19] \\
\hline 1-pentanol & 3.6681 & 3.4350 & 246.0 & 0.0100 & 2233.5 & 1.70 & 0.029 & -0.034 & 0.610 & 0.625 & 251 & 586 & [19] \\
\hline
\end{tabular}
The objective function given by equation (8) was minimized by the Levenberg-Marquardt algorithm.

$$
\sum_{i}^{2 \mathrm{~N}}\left(\frac{\Omega_{\mathrm{ref}, i}-\Omega_{\mathrm{cal}, i}}{\Omega_{\mathrm{ref}, i}}\right)^{2}=\min
$$

Table 1. PCP-SAFT molecular parameters for selected alkanols and deviations from the reference data 
General quantity $\Omega$ stands either for the vapor pressure $p$ or the saturated liquid density $\rho_{\mathrm{L}}$. $2 \mathrm{~N}$ denotes a sum of the correlated points for $p$ and $\rho_{\mathrm{L}}$. Values for the PCP-SAFT parameters together with the dipole moment $\mu$ taken from CRC Handbook of Chemistry and Physics [17] are summarized in Table 1. The average deviation $A D$ and standard deviation $S D$ of the PCP-SAFT EoS from the reference data are also provided in Table 1. $A D$ and $S D$ are defined in terms of equations (9) and (10), respectively.

$$
\begin{gathered}
A D=\frac{1}{N} \sum_{i=1}^{N} \frac{\Omega_{\mathrm{ref}, i}-\Omega_{\mathrm{cal}, i}}{\Omega_{\mathrm{ref}, i}} \cdot 100 \\
S D=\sqrt{\frac{1}{N} \sum_{i=1}^{N}\left(\frac{\Omega_{\mathrm{ref}, i}-\Omega_{\mathrm{ca}, i}}{\Omega_{\mathrm{ref}, i}} \cdot 100\right)^{2}-A D^{2}}
\end{gathered}
$$

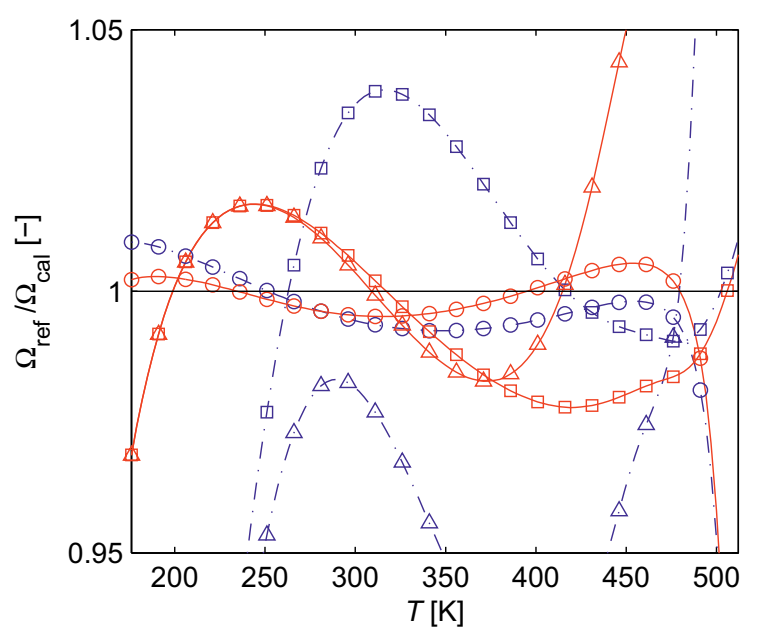

Figure 1. Deviation of the PCP-SAFT EoS (red solid line) and the PC-SAFT EoS (blue dash-dotted line) from the reference data for methanol [18]; $\square$, vapor pressure $(p)$; o, saturated liquid density $\left(\rho_{\mathrm{L}}\right) ; \Delta$, saturated vapor density $\left(\rho_{\mathrm{V}}\right)$

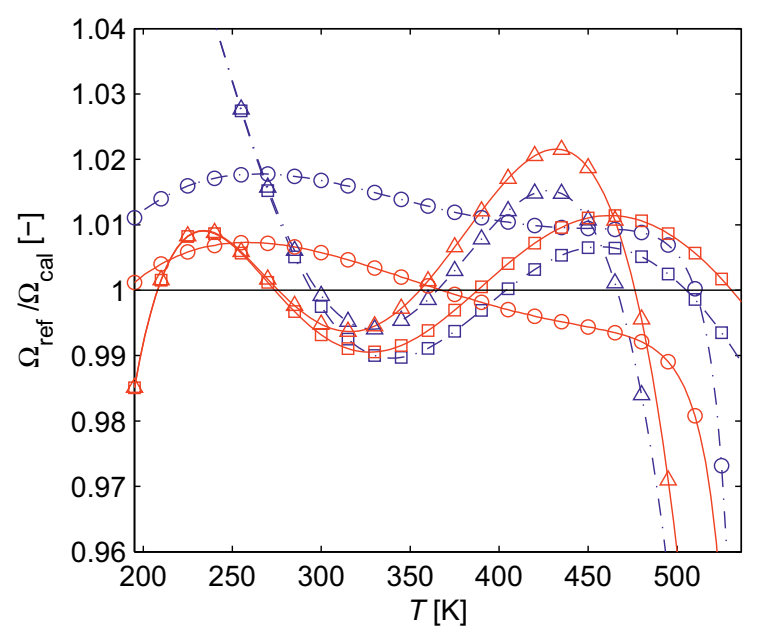

Figure 2. Deviation of the PCP-SAFT EoS (red solid line) and the PC-SAFT EoS (blue dash-dotted line) from the reference data for 1-propanol [19]; $\square$, vapor pressure $(p)$; o, saturated liquid density $\left(\rho_{\mathrm{L}}\right) ; \Delta$, saturated vapor density $\left(\rho_{\mathrm{V}}\right)$
The saturated liquid density was correlated only up to reduced temperature $T_{\mathrm{r}}=T / T_{\text {crit }}=0.95$, while the vapor pressure was fitted almost up to the critical point, i.e. to reduced temperature of $\sim 0.999$. Deviation of both the PCP-SAFT EoS and the PC-SAFT EoS from the reference saturation conditions depending on temperature is given in figures 1 and 2 for methanol and 1-propanol, respectively. The accuracy of the saturation conditions predicted by both EoSs is almost the same for all investigated substances. The PCP-SAFT EoS gives slightly better results, especially, for the vapor pressure.

Figure 3 shows $\log (p v)$ diagram for ethanol. The PCPSAFT prediction is compared with the data taken from the NIST reference database REFPROP [18] and results from the Soave-Redlich-Kwong cubic EoS. Only some of the reference data used in the Levenberg-Marquardt algorithm is plotted in the figure for better representation.

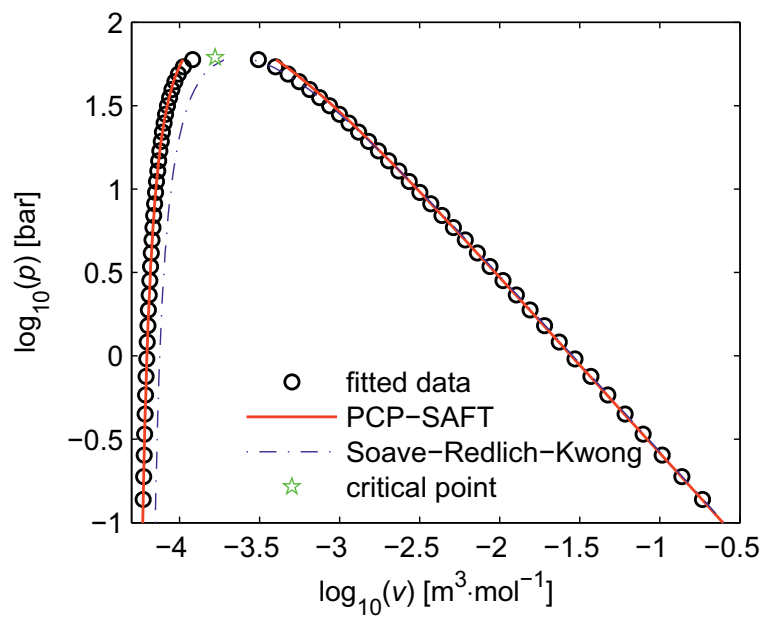

Figure 3. Comparison of the PCP-SAFT EoS, Soave RedlichKwong cubic EoS and the reference data [18] in $\log (p v)$ diagram for ethanol

\section{Density gradient theory}

The Cahn-Hilliard density gradient theory $[1,20,21]$ can successfully be applied on planar [22,23] and also on spherical phase interfaces $[24,25]$. In GT, the following Euler-Lagrange equation has to be satisfied by the interfacial density profile

$$
\sum_{j} \nabla\left(c_{i j} \nabla \rho_{j}\right)-\frac{1}{2} \sum_{j, k} \frac{\partial c_{j k}}{\partial \rho_{i}} \nabla \rho_{j} \nabla \rho_{k}=\frac{\partial \omega}{\partial \rho_{i}},
$$

where $c_{i j}$ denotes the influence parameter describing the effect of the density gradients on the local Helmholtz energy and $\omega$ is the grand-potential density for a hypothetical homogeneous fluid [21,23]. The grand potential density needs to be determined from a convenient EoS in GT. The employed fluid model has to provide a physically reasonable solution in the metastable region along the van der Waals isotherm which excludes application of the highly accurate semi-empirical EoSs. SAFT-type EoSs represent a good improvement compared to cubic EoSs employed within GT in the past $[22,25]$. 


\subsection{Planar phase interface}

For a planar phase interface, equation (11) can be simplified in the following form [21]

$$
\sum_{i, j} \frac{1}{2} c_{i j} \frac{\mathrm{d} \rho_{j}}{\mathrm{~d} z} \frac{\mathrm{d} \rho_{i}}{\mathrm{~d} z}=\omega(\rho)-\omega^{0} \equiv \Delta \omega,
$$

where $\omega^{0}=-p^{0}$ is the grand-potential density of the homogeneous vapor. After some modifications [21,22] of equation (12), the surface tension at the planar phase interface of a pure fluid can be determined

$$
\sigma=\int_{\rho_{\mathrm{V}}}^{\rho_{\mathrm{L}}} \sqrt{2 c \Delta \omega(\rho)} \mathrm{d} \rho .
$$

\subsection{Influence parameter}

A constant, i.e. temperature and density independent, influence parameter $c$ was considered in this study. Values for $c$ were evaluated by correlating equation (13) to available surface tension data. Table 2 summarizes average values for $c$ both for PCP-SAFT, i.e. by considering dipolar moments of alkanols, and for original PC-SAFT [11] without polar term in equation (2).

Table 2. Values of the influence parameter $(c)$ for PCP-SAFT and PC-SAFT with and without the polar effect, respectively.

\begin{tabular}{llrl}
\hline \multirow{2}{*}{ Substance } & \multicolumn{2}{c}{$c\left[\mathbf{J}^{\mathbf{5}} \mathbf{m}^{\mathbf{5}} \mathbf{m o l}^{-\mathbf{2}}\right]$} & \multirow{2}{*}{ Ref. } \\
& PCP-SAFT & PC-SAFT $^{\mathrm{a}}$ & \\
\hline methanol & $2.4525 \mathrm{E}-20$ & $3.2293 \mathrm{E}-20$ & {$[18,26]$} \\
ethanol & $5.1039 \mathrm{E}-20$ & $5.4534 \mathrm{E}-20$ & {$[18]$} \\
1-propanol & $8.6837 \mathrm{E}-20$ & $9.0175 \mathrm{E}-20$ & {$[26]$} \\
2-propanol & $8.0856 \mathrm{E}-20$ & $8.2775 \mathrm{E}-20$ & {$[27,28]$} \\
1-butanol & $1.4645 \mathrm{E}-19$ & $1.5387 \mathrm{E}-19$ & {$[29]$} \\
1-pentanol & $2.3080 \mathrm{E}-19$ & $2.3054 \mathrm{E}-19$ & {$[26]$} \\
\hline
\end{tabular}

${ }^{\text {a }}$ For the original PC-SAFT molecular parameters [11]

Surface tension of most of the selected substances can be well approximated by the Somayajulu's [26] correlation valid over wide temperature ranges. Mulero et al. [29] have recently revisited and improved this correlation for some fluids, e.g., 1-butanol. The influence parameter for ethanol was evaluated from the REFPROP [18] data, using recent correlation by Mulero et al. [30], instead of the Somayajulu's [26] correlation. The REFPROP data seems to be more accurate, especially in the central temperature region, as it agrees well with another recent correlation by Goncalves et al. [31]. On the other hand, no such discrepancy between the REFPROP data and the Somayajulu's correlation was found in case of methanol.

\section{Surface tension of associating fluids}

The surface tension of a pure substance can be calculated from equation (13). Several examples of the temperature dependency of the surface tension calculated both by
GT + PCP-SAFT, i.e. by considering nonzero dipolar moments of the alkanol molecules, and by GT + PCSAFT, i.e. with neglected polar term, are shown in this section. The results of both models are compared with the reference data for the surface tension.

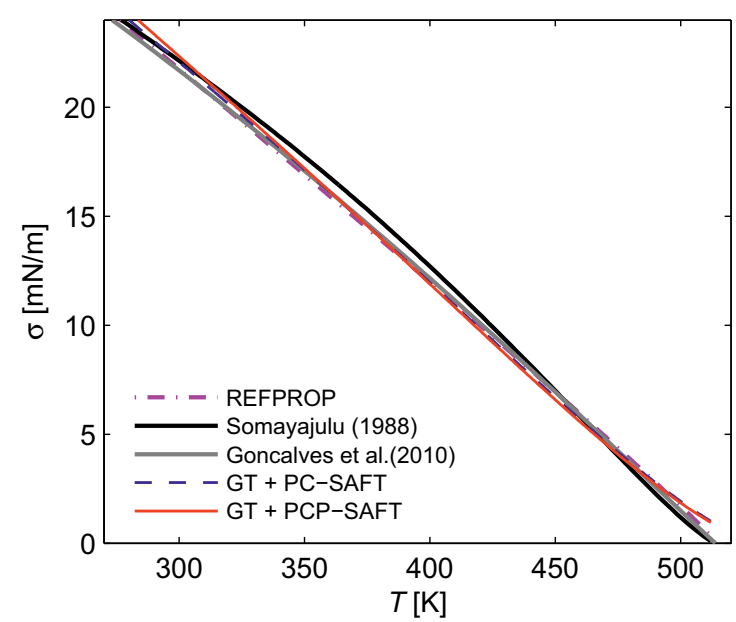

Figure 4. Surface tension predicted by GT + PC-SAFT and GT + PCP-SAFT for ethanol; comparison with the data from REFPROP [18], and the temperature correlations by Somayjulu [26] and Goncalves et al. [31]

Figure 4 shows temperature dependence of the surface tension for ethanol. As can be seen, both GT + PC-SAFT and GT + PCP-SAFT provide quite good and comparable results over wide temperature range exceeding $200 \mathrm{~K}$. The modeled surface tension agrees well both with the REFPROP [18] data and the correlation by Congalves et al. [31]. Somayajulu's [26] correlation provides moderately higher surface tension within the central temperature region. An interesting result is that the GT + PC-SAFT model gives a slightly better prediction at lower temperatures in this case. Consequently, consideration of the ethanol as a dipolar substance did not bring any improvement in the predicted surface tension.

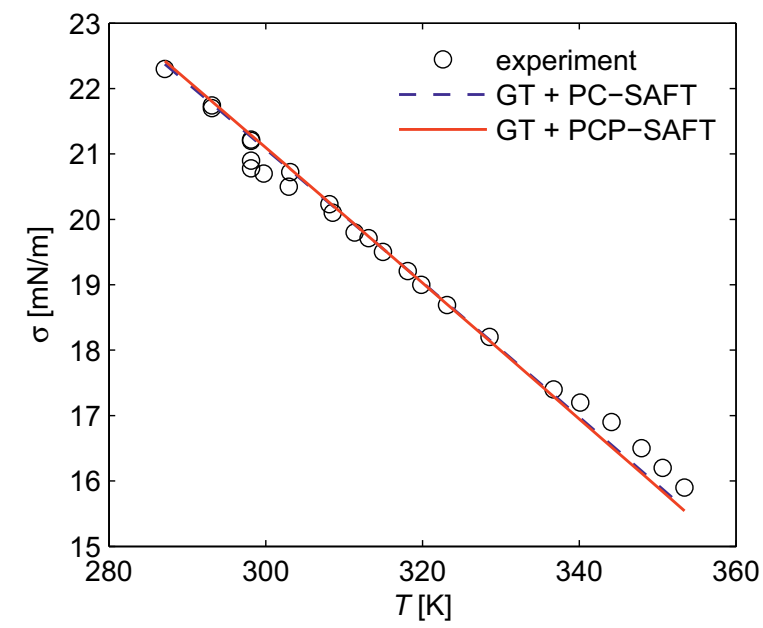

Figure 5. Surface tension predicted by GT + PC-SAFT and GT + PCP-SAFT compared to the experimental data $[27,28]$ for 2-propanol

Another example is given in figure 5 for 2-propanol. The predicted results are compared to the experimental 
data by Hoke and Chen [27] and Vasquez et al. [28]. As can be seen, both approaches provide almost the same surface tension in the considered temperature range. Moreover, both models are in good agreement with the experimental data in this case.

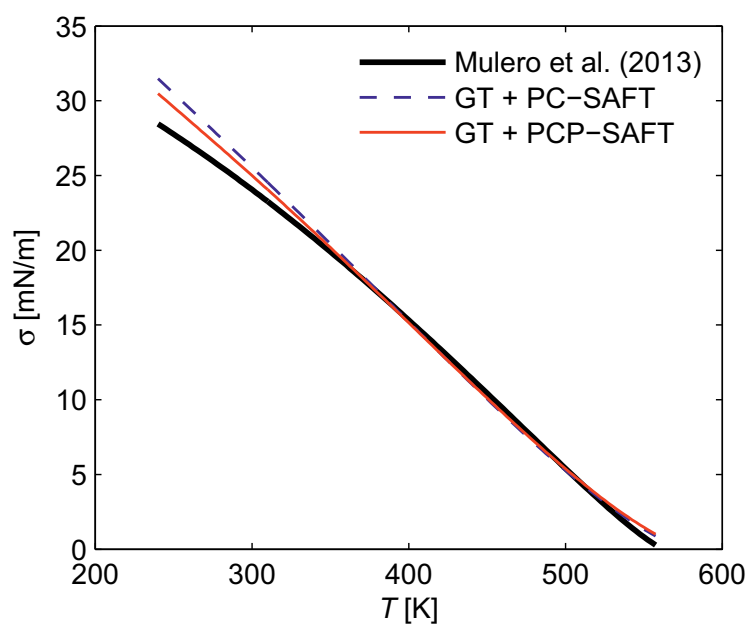

Figure 6. Surface tension predicted by GT + PC-SAFT and GT + PCP-SAFT compared to the temperature correlation by Mulero et al. [29] for 1-butanol

Figure 6 compares GT + PC-SAFT and GT + PCPSAFT with the surface tension correlation by Mulero et al. [29] for 1-butanol. The GT + PCP-SAFT model gives slightly better results in this case. Nevertheless, both models differ from the correlation by Mulero et al. at the low temperature region. The reason is that the influence parameter for alkanols varies rather strongly with temperature. Consequently, considering a constant value for $c$ resulted in discrepancies between the modeled surface tension and the reference data.

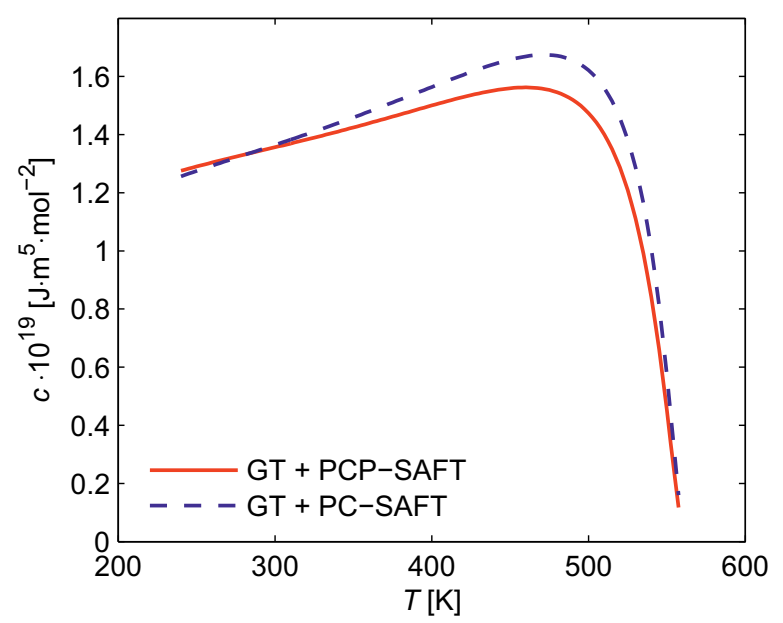

Figure 7. Influence parameter for GT + PCP-SAFT and GT + PC-SAFT for 1-butanol fitted to the surface tension correlation by Mulero et al. [29]

As can be seen in figure 7, application of PCP-SAFT resulted in only slight a improvement in the shape of temperature dependency of the influence parameter compared to the non-polar PC-SAFT EoS.
Unlike in the previous study [23], PCP-SAFT implemented in GT gives for the selected alkanols almost identical results as GT + non-polar PC-SAFT. The polar interactions given by term $F_{\text {pol }}$ in equation (2) have much smaller influence than the association strengths $F_{\text {as }}$ for the investigated substances.

\section{Conclusion}

The density gradient theory combined with two SAFTtype equations of state was used to model surface tension of associating dipolar fluids. Both GT + PC-SAFT and GT + PCP-SAFT give reasonable prediction for surface tension of selected alcohols.

Results of the non-polar PC-SAFT EoS implemented in GT are comparable to the surface tension obtained with GT + PCP-SAFT comprising the dipolar effects. In contrary to our previous study [23] focused on nonassociating polar components such as carbon dioxide or halogenated hydrocarbons, the consideration of the dipolarity for the selected associating fluids, i.e. shortchain normal alkanols, did not bring any significant improvement in the predicted surface tension. The temperature dependence of the influence parameter reflecting convenience of the equation of state employed within GT - was almost the same for both non-polar and polar models for all investigated substances.

Other associating dipolar fluids such as amines and associating quadrupolar-dipolar substances, e.g., hydrogen sulfide, will also be investigated in future.

\section{Acknowledgement}

The study has been supported by grants from the Czech Science Foundation No.: GPP101/11/P046, the Academy of Sciences of the Czech Republic No.: IAA200760905, M100761201, and from the institutional support RVO:61388998. Many thanks go also to E.W. Lemmon from NIST, Boulder, for his help with evaluation of the surface tension for ethanol using REFPROP.

\section{References}

1. J. W. Cahn, J. E. Hilliard, J. Chem. Phys. 28, 258 (1958)

2. J. Gross, G. Sadowski, Ind. Eng. Chem. Res. 40, $1244(2001)$

3. E. A. Müller, K. E. Gubbins, Ind. Eng. Chem. Res. 40, 2193 (2001)

4. V. Vinš, V. Vacek, J. Chem. Eng. Data 54, 2395 (2009)

5. G. Hallewell, V. Vacek, V. Vinš, Fluid Phase Equil. 292, 64 (2010)

6. J. Gross, O. Spuhl, F. Tumakaka, G. Sadowski, Ind. Eng. Chem. Res. 42, 1266 (2003)

7. J. Gross, AIChE J. 51, 2556 (2005)

8. V. Vinš, J. Hrubý, Int. J. Refrigeration 34, 2109 (2011)

9. A. Grenner, G. M. Kontogeorgis, N. von Solms, M. L. Michelsen, Fluid Phase Equil. 258, 83 (2007)

10. X. Tang, J. Gross, Fluid Phase Equil. 293, 11 (2010) 
11. J. Gross, G. Sadowski, Ind. Eng. Chem. Res. 41, 5510 (2002)

12. W. G. Chapman, K. E. Gubbins, G. Jackson, M. Radosz, Ind. Eng. Chem. Res. 29, 1709 (1990)

13. S. H. Huang, M. Radosz, Ind. Eng. Chem. Res. 29, 2284 (1990)

14. S. H. Huang, M. Radosz, Ind. Eng. Chem. Res. 30, 1994 (1991)

15. J. Gross, J. Vrabec, AIChE J. 52, 1194 (2006)

16. J. Vrabec, J. Gross, J. Phys. Chem. B 112, 51 (2008)

17. D. R. Lide: CRC Handbook of Chemistry and Physics, Cleveland, USA, 2004

18. E.W. Lemmon, M.L. Huber, M.O. McLinden, REFPROP, NIST Standard Reference Database 23 Version 9.1 (2013)

19. T.E. Daubert, R.P. Danner, H.M. Sibul, C.C. Stebbins: Physical and Thermodynamic Properties of Pure Chemicals: Data Compilation; Taylor \& Francis: Washington DC (1989)

20. J. W. Cahn, J. Chem. Phys. 30, 1121 (1959)

21. H. T. Davis, L. E. Scriven, Advances in Chemical Physics 49, 357 (1982)

22. V. Vinš, J. Hrubý, B. Planková, EPJ Web Conf. 25, 02028 (2012)

23. V. Vinš, B. Planková, J. Hrubý, Int. J. Thermophys. 34, 792 (2013)

24. B. Planková, J. Hrubý, V. Vinš, EPJ Web Conf. 45, 01076 (2013)

25. J. Hrubý, D. G. Labetski, M. E. H. van Dongen, J. Chem. Phys. 127, 164720 (2007)

26. G. R. Somayajulu, Int. J. Thermophys. 9, 559 (1988)

27. B.C. Hoke, J.C. Chen: J. Chem. Eng. Data 36, 322 (1991)

28. G. Vazquez, E. Alvarez, J.M. Navaza, J. Chem. Eng. Data 40, 611 (1995)

29. A. Mulero, M.I. Parra, I. Cachadina, Fluid Phase Equil. 339, 81 (2013)

30. A. Mulero, I. Cachadina, M.I. Parra, J. Phys. Chem. Ref. Data 41, 043105 (2012)

31. F.A.M.M. Goncalves, A.R. Trindade, C.S.M.F. Costa, J.C.S. Bernardo, I. Johnson, I.M.A. Fonseca, A.G.M. Ferreira, J. Chem. Thermodynamics 42, 1039 (2010) 